\title{
Cardiac injury as prognostic value in COVID-19: more remains to be clarified
}

\author{
Nicola Mumoli ${ }^{1} \cdot$ Marco Cei $^{2} \cdot$ Antonino Mazzone $^{1} \cdot$ Giulia Conte $^{1}$
}

Received: 4 October 2020 / Accepted: 12 October 2020 / Published online: 28 October 2020

(c) Società Italiana di Medicina Interna (SIMI) 2020

Coronavirus disease 2019 (COVID-19) is a newly recognized infectious disease, caused by the severe acute respiratory syndrome Coronavirus 2 (SARS-CoV-2), with a high rate of morbidity and mortality $[1,2]$. In particular, with COVID-19 infection, mortality rates are highest in older patients $(14.8 \%$ in those $>80$ years) and in patients with cardiovascular disease. Currently, there is no evidencebased treatment for COVID-19, consequently, the mainstay of treatment is supportive care [3]. Acute respiratory distress syndrome (ARDS) is considered to be an acute process confined to the lungs, with an overall case-fatality rate for COVID 19 which varies between $1.4 \%$ and $2.3 \%$. However, there is more and more evidence which identifies myocardial injury as COVID-19 related-complication, manifesting as left ventricular dysfunction and troponin elevation, with an incidence ranging from $7.2 \%$ to $12 \%$ [4]. Nevertheless, while an American College of Cardiology clinical bulletin pointed out the cardiac implications of COVID-19, the link between COVID-19-associated cardiac injury and risk of mortality remains unclear. Recent studies have highlighted the association between myocardial injury and fatal outcomes of COVID-19 disease. Particularly, inflammation caused by SARS-COV 2 appears to result in myocardial injury, leading to impairment of cardiac function, and increased incidence of ventricular tachyarrhythmias. Conversely, the prognosis of patients without myocardial injury appears relatively favorable, even for those with underlying cardiovascular disease. Patients with myocardial injury have elevated levels of myocardial markers, such as circulating cardiac troponin, a marker related but not limited to myocardial injury. In

Nicola Mumoli

nimumoli@tiscali.it

1 Department of Internal Medicine, ASST Ovest Milanese, Ospedali di Magenta e Legnano, Ospedale Fornaroli, via al Donatore di Sangue 50, 20013 Magenta, MI, Italy

2 Department of Internal Medicine, Cecina Hospital, Cecina, LI, Italy particular, troponin elevation, during COVID-19 infection, is likely to be multifactorial and it is not only attributable to atherothrombotic coronary occlusion. For example, in critically unwell patients, the oxygen supply-demand occurs at a cellular level in the majority of organ systems, but the sensitivity of cardiac troponin testing substantially is one of the earliest and most precise indicators of end-organ dysfunction, thus allowing an early start of the oxygen supplement to improve tissue oxygenation and perfusion. Moreover, the elevation of troponin in this kind of patients seems to play a negative prognostic role. In a cohort study of 191 patients with confirmed COVID-19 infection, the elevation of troponin was closely related to mortality increase [5]. In another study, the number of patients with an elevation of troponin concentration was more likely to require invasive or noninvasive ventilation and to develop acute respiratory distress syndrome or acute kidney injury [1]. Nevertheless, data about the clinical significance of cardiac injury in noncritically ill COVID-19 patients and about the correlation between cardiac injury and mortality are still lacking and unclear. In particular, the interaction between heart damage, comorbidity, and mortality is not well defined.

In this issue, Cipriani et al. [6], reported the results of a single-center, observational study to describe the clinical characteristics, the incidence of cardiac injury and outcomes in a cohort of Italian COVID-19 patients admitted to a medical non-intensive COVID-19 Unit. Furthermore, they performed a post-hoc exploratory mediation analysis to evaluate the relationship between high-intensity cardiac troponin I (Hs cTnI) and in-hospital mortality. During the study period, a total of 109 COVID-19 inpatients (female 36\%, median age 71 years) were enrolled, with no-need or no-eligibility for age or comorbidities for mechanical or non-invasive ventilation. Twenty-seven patients without troponin testing were excluded. 20 patients (18\%) died in-hospital and, compared with survivors, these patients were older and had more comorbidities defined by Charlson comorbidity index $=/>3$ ( $65 \%$ vs $24 \%, p=0.001)$. Regarding laboratory parameters, 
dead patients presented higher levels of Hs-cTnI, both at first evaluation and peak levels. A dose-response curve between Hs-cTnI and in-hospital mortality risk up to $200 \mathrm{ng} / \mathrm{L}$ was detected. The strongest mediator of mortality was peak HscTnI, followed by chronic kidney disease, and chronic coronary artery disease (CAD). Smaller effects were observed for age, lactic dehydrogenase (LDH), and D-dimer.

There are some limitations in this study, mainly related to be a single center, with a small sample-size, retrospective and observational study. Furthermore, patients enrolled in this study did not require non-invasive ventilation or were not considered suitable for age or comorbidity. Therefore, the data obtained may not apply to all patients with COVID19 infection, especially the most critically ill. The authors choose not to analyze data from electrocardiography and cardiac imaging, mainly to avoid virus spreading when patients had to move out of the ward. Avoiding tests unnecessary or redundant for the management is certainly ethically sound; however, this lessens the strength of interpretation of troponin elevation that remained confined to aspecific "myocardial damage". This fact highlights the difference between retrospective and well-designed prospective trials, in which rigorous definitions of the cardiac spectrum of disease (i.e., myocardial infarctions, Tako-tsubo syndrome or myocarditis versus minimal myocardial damage) required appropriate diagnostic work-up.

This is a strategic aspect when the study aims to clarify a gray zone in knowledge.

In conclusion, Cipriani et al. confirmed the role of Hs$\mathrm{cTnI}$ as the most important predictor of in-hospital mortality also in non-critically ill patients with COVID-19, and the central role of cardiac damage in the prognosis of patients with COVID-19 infection. Therefore, as well as in other pathologies (for example pulmonary thromboembolism), troponin elevation is related to an increased risk of shortterm mortality and conditioning the possible use of more intensive care.

Although the elevation troponin is the strongest predictor of in-hospital mortality, in this study, other factors were found to be predictors of in-hospital mortality such as chronic kidney disease, CAD, age, and elevation of LDH and D-dimer. Shi et al. [1] found that markers of the inflammatory response, such as $\mathrm{C}$-reactive protein, procalcitonin, and leukocytes, were significantly increased among patients who suffered from cardiac injury and therefore more at risk of short-term mortality. In another study [4] patients with COVID-19 who were admitted to the intensive care unit had higher plasma levels of cytokines, such as interleukin (IL)-2 and tumor necrosis factor-alpha. To better identify patients with a higher risk of short-term mortality, the use of a score that includes factors mostly associated with poor prognosis could be conceivable.

Funding None.

\section{Compliance with ethical standards}

Conflict of interest The authors declare that they have no conflict of interest.

Statement of human and animal rights This article does not contain any studies with human participants or animals performed by any of the authors.

Informed consent None.

\section{References}

1. Shi S, Qin M, Shen B, Cai Y, Liu T, Yang F, Gong W, Liu X, Liang J, Zhao Q, Huang H, Yang B, Huang C (2020) Association of cardiac injury with mortality in hospitalized patients with COVID-19 in Wuhan, China. JAMA Cardiol 5(7):802-810

2. Guo T, Fan Y, Chen M, Wu X, Zhang L, He T, Wang H, Wan J, Wang X, Lu Z (2020) Cardiovascular implications of fatal outcomes of patients with coronavirus disease 2019 (COVID-19). JAMA Cardiol 5(7):811-818

3. Chapman AR, Bularga A, Mills NL (2020) High-sensitivity cardiac troponin can be an ally in the fight against COVID-19. Circulation 141(22): 1733-1735

4. Shi S, Qin M, Cai Y, Liu T, Shen B, Yang F, Cao S, Liu X, Xiang Y, Zhao Q, Huang H, Yang B, Huang C (2020) Characteristics and clinical significance of myocardial injury in patients with severe coronavirus disease 2019. Eur Heart J 41(22):2070-2079

5. Zhou F, Yu T, Du R, Fan G, Liu Y, Liu Z, Xiang J, Wang Y, Song B, Gu X, Guan L, Wei Y, Li H, Wu X, Xu J, Tu S, Zhang Y, Chen H, Cao B (2020) Clinical course and risk factors for mortality of adult inpatients with COVID-19 in Wuhan, China: a retrospective cohort study. Lancet 395(10229):1054-1062

6. Cipriani A, Capone F, Donato F, Molinari L, Ceccato D, Saller A, Previato L, Pesavento R, Sarais C, Fioretto P, Iliceto S, Gregori D, Avogaro A, Vettor R (2020) Cardiac injury and mortality in patients with Coronavirus disease 2019 (COVID-19): insights from a mediation analysis. Intern Emerg Med. https:// doi.org/10.1007/s11739-020-02495-w

Publisher's Note Springer Nature remains neutral with regard to jurisdictional claims in published maps and institutional affiliations. 